\title{
OBTENÇÃO DE GÁS CARBÔNICO NO ENSINO MÉDIO: UMA PRÁTICA EXPERIMENTAL PARA DEBATER O TEMA AQUECIMENTO GLOBAL
}

\author{
Renato Nunes de Andrade1, Rayza Rosa Tavares Rodrigues2, \\ Naiane Nunes Gonçalves ${ }^{3}$, Bruno Magela de Melo Siqueira4, Rafael Baioco Ruy ${ }^{5 *}$ \\ Secretaria de Estado de Educação do Espírito Santo \\ Escola Estadual de Ensino Médio Professor Renato José da Costa Pacheco. Vitória - ES.
}

\begin{abstract}
RESUMO
A temática "Gases", no contexto do efeito estufa, foi desenvolvida em aulas experimentais de química do Ensino Médio, visando a preparação para o Exame Nacional do Ensino Médio ENEM. Monitorados por um professor de química, alunos do curso de licenciatura em química planejaram aulas práticas, que foram ministradas no laboratório de ciências da escola estadual com uso de materiais alternativos e industrializados. Oito turmas do $2^{\mathrm{o}}$ ano do ensino médio participaram deste estudo. A estratégia de ensino produziu a articulação dos conteúdos programáticos trabalhados em sala de aula de forma teórica com a prática, a partir das observações e as evidências dos resultados experimentais obtidos pelos alunos do ensino médio.
\end{abstract}

Palavras-chave: ensino de química, efeito estufa, aquecimento global, gás carbônico.

\begin{abstract}
The theme "gases" in the context of global warming, has been developed in experimental chemistry classes of high school, in order to prepare for the National High School Exam of Brazil - ENEM. Monitored by a professor of chemistry, students of planned degree in chemistry practical classes, which were taught in the science laboratory of state school with use of alternative and industrial materials. Eight classes of 2 nd year high school participated in this study. The teaching strategy produced the articulation of the syllabus worked in the classroom in a theoretical way with the practice, from the observations and evidence from experimental results obtained by high school students.
\end{abstract}

Keywords: greenhouse effect, global warming, carbon dioxide.

\footnotetext{
1 aluno do curso de Licenciatura em Química do IFES - Campus Vila Velha. E-mail: a_programador@hotmail.com.

2 aluna do curso de Licenciatura em Química do IFES - Campus Vila Velha. E-mail: rayzartr@hotmail.com

${ }^{3}$ aluna do curso de Licenciatura em Química do IFES - Campus Vila Velha. E-mail: naiane.nng@hotmail.com

4 aluno do curso de Licenciatura em Química do IFES - Campus Vila Velha. E-mail: brunosiq_es@hotmail.com

5 professor de Química, M.Sc. EEEM Professor Renato José da Costa Pacheco. E-mail: rbaioco@yahoo.com.br
} 


\section{INTRODUÇÃO}

A demanda pela produção de energia cresce em ritmo proporcional ao desenvolvimento tecnológico. A produção de energia dá-se principalmente por métodos tradicionais, como, por exemplo, a queima de combustíveis fósseis, pois essas técnicas são altamente lucrativas. Este tipo de atividade traz como resultado a emissão de gases do efeito estufa. Outras fontes podem contribuir para emissão de gases que retém o calor, mas o gás tratado neste trabalho será o dióxido de carbono.

O aquecimento global é causado pela intensificação do efeito estufa na atmosfera. O efeito estufa é um fenômeno natural importante, pois permite que a Terra se mantenha aquecida a uma temperatura adequada à sobrevivência de diversos organismos. No entanto, a ação do homem o tem tornado cada vez mais intenso, provocando aumento da temperatura do planeta. O principal responsável pela intensificação do efeito estufa são as emissões de dióxido de carbono na atmosfera, causadas principalmente pelas indústrias, queimadas e veículos automotores.

Quando os raios solares atingem a atmosfera e a superfície da Terra, cerca de $70 \%$ da energia é absorvida pelo solo, pelos oceanos, pelas plantas, entre outras superfícies. Os demais 30\% são refletidos para o espaço por nuvens e outras superfícies refletivas. No entanto, o calor absorvido é posteriormente irradiado para fora do planeta, sendo nesta etapa que os gases responsáveis pelo efeito estufa entram em ação. Os gases dióxido de carbono, metano, vapor d'água, entre outros gases, são responsáveis por manter parte do calor irradiado retido na atmosfera. $O$ calor que não consegue sair pela atmosfera da Terra mantém o planeta mais aquecido do que o espaço sideral. O efeito estufa é gerado pelas queimadas das florestas, pois a vegetação regula a temperatura terrestre, os ventos e o nível de chuvas em diversas regiões. Como as florestas estão diminuindo no mundo, a temperatura terrestre tem aumentado.

Outro fator que está intensificando o efeito estufa é o lançamento de gases poluentes na atmosfera, principalmente os que resultam da queima de combustíveis fósseis. A queima do óleo diesel e da gasolina nos grandes centros urbanos tem colaborado para o aumento do efeito estufa. O dióxido de carbono e o monóxido de carbono ficam concentrados em determinadas regiões da atmosfera formando uma camada que bloqueia a dissipação do calor. Outros gases que contribuem para este 


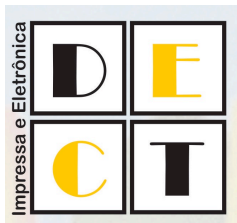

processo são o gás metano e óxidos de nitrogênio. Esta camada de poluentes, tão presente nas grandes cidades, funciona como um isolante térmico do planeta Terra. $\mathrm{O}$ calor fica retido nas camadas mais baixas da atmosfera trazendo graves problemas ao planeta.

Figura 1. Esquema geral do efeito estufa no planeta Terra.

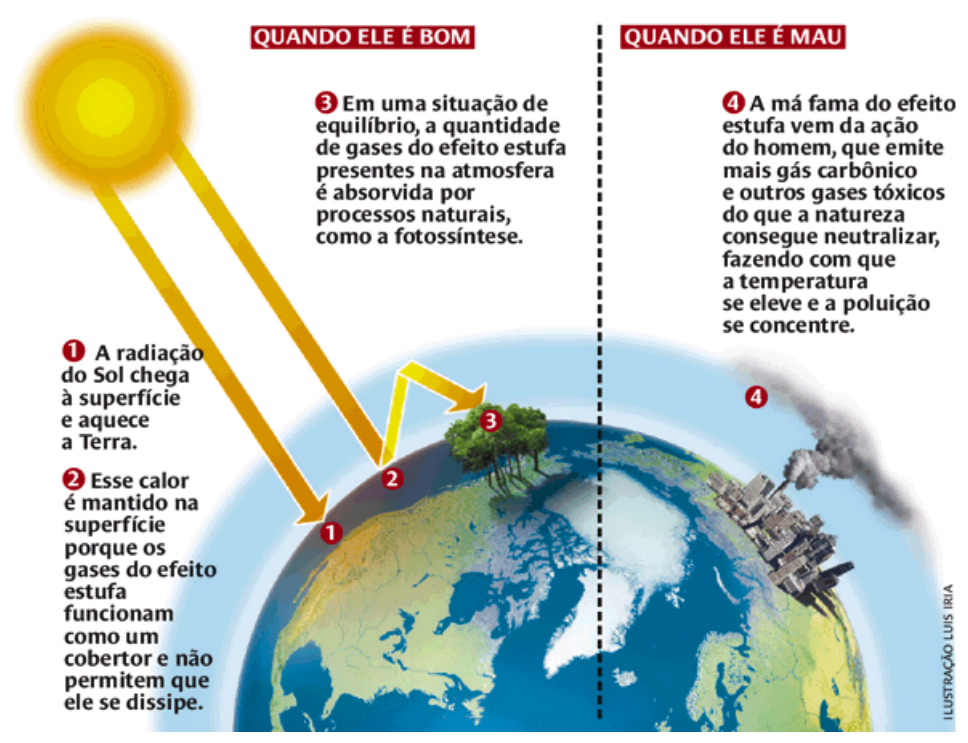

Fonte: http://soumaisenem.com.br/geografia/o-meio-ambiente/aquecimento-global.

Este trabalho foi desenvolvido na Escola Estadual de Ensino Médio Professor Renato José da Costa Pacheco, em parceria com bolsistas do PIBID de Química do Instituto Federal do Espírito Santo, campus de Vila Velha, e buscou desafiar o senso crítico dos alunos por meio de aulas práticas relacionadas à obtenção do principal agente do aquecimento global, o gás carbônico. Foram discutidos os meios pelos quais acontecem as emissões atmosféricas e alternativas para reduzir tais emissões, os efeitos da circulação de gás carbônico na atmosfera e a necessidade de se investir em fontes de energia limpa e renovável.

Apesar dos alunos não estarem na última série do ensino básico, prepará-los para o ENEM se faz necessário, visto que os conteúdos são cumulativos e subsequentes. Fornecer aulas práticas que fogem da rotina das aulas expositivas teóricas contribui para estimular o interesse e comprometimento pela disciplina, além de ser uma importante ferramenta de propagação do ensino, pois acarreta uma melhor assimilação dos conteúdos, "pois a química é uma ciência experimental, fica por isso muito difícil aprende-la sem a realização de atividades práticas” (SILVA, 2011). 


\section{PROCEDIMENTOS METODOLÓGICOS}

As aulas foram apresentadas para oito turmas de alunos do $2^{\circ}$ ano do ensino médio, tiveram duração de 55 minutos e foram divididas em três etapas, conforme orientações do roteiro fornecido aos alunos. Na primeira etapa houve apresentação de uma introdução contextualizada focada no tema efeito estufa, assim como apresentada na introdução deste trabalho, e os alunos foram estimulados a desenvolver o senso crítico sobre o tema.

$\mathrm{Na}$ segunda etapa os alunos foram convidados a participar da secção experimental. Para tal, foi montado um aparato similar ao descrito pela Figura 2. Os materiais e reagentes utilizados foram carbonato de cálcio, ácido muriático, água, corante, balança semi-analítica, espátula, kitasato, proveta, bureta, termômetro, bacia de vidro, rolha de borracha e mangueira conectora.

Figura 2. Aparato usado na obtenção de gás carbônico.

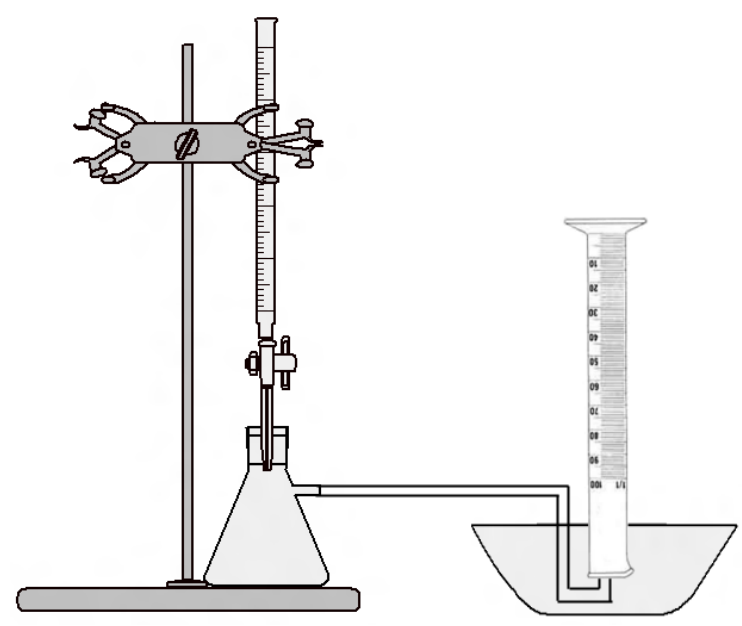

A obtenção do gás carbônico deu-se através da reação entre o carbonato de cálcio e o ácido clorídrico comercial, conforme Reação 1.

$\mathrm{CaCO}_{3(\mathrm{~s})}+2 \mathrm{HCl}_{(\mathrm{aq})} \rightarrow \mathrm{CaCl}_{2(\mathrm{aq})}+\mathrm{H}_{2} \mathrm{O}_{(\mathrm{g})}+\mathrm{CO}_{2(\mathrm{~g})}$ 
Os procedimentos foram elaborados de maneira que o volume teórico de gás carbônico produzido fosse de $450 \mathrm{~mL}$, uma vez que se utilizou uma proveta de $500 \mathrm{~mL}$ invertida para se coletar o gás produzido. Utilizando o conhecimento das relações estequiométricas envolvidas na reação e do estudo dos gases, em que a equação para os gases ideais, $\mathrm{pV}=\mathrm{nRT}$, foi empregada, foram propostas as seguintes variáveis para obtenção do gás carbônico:

Pressão: $\mathrm{p}=1 \mathrm{~atm}$

Volume: $\mathrm{V}=0,450 \mathrm{~L}$

Número de mol de $\mathrm{CO}_{2}: \mathrm{n}=0,0184$ mol, equivalente a $1,843 \mathrm{~g}$ de $\mathrm{CaCO}_{3}$

Constante universal dos gases: $\mathrm{R}=0,082 \frac{\mathrm{atm} \mathrm{L}}{\mathrm{K} \mathrm{mol}}$

Temperatura: $\mathrm{T}=298 \mathrm{~K}$, equivalente a $25^{\circ} \mathrm{C}$

Para execução do experimento, uma massa de aproximadamente $1,843 \mathrm{~g}$ de $\mathrm{CaCO}_{3}$ foi medida e transferida quantitativamente para o kitassato. A bureta foi completamente preenchida com ácido clorídrico comercial e acoplada ao kitassato. Uma quantidade em excesso de ácido clorídrico foi adicionada a amostra de $\mathrm{CaCO}_{3}$ resultando na ocorrência da Reação 1 . O gás carbônico obtido pela reação foi quantitativamente transferido para proveta invertida através de uma mangueira conectora. $\mathrm{Na}$ terceira etapa, os alunos compararam o volume de gás carbônico obtido experimentalmente com valor esperado teoricamente. Os resultados teóricos e experimentais foram confrontados por meio de tratamento estatístico em que foi calculado a média, o erro relativo e o desvio padrão da média. Também houve discussões sobre precisão e exatidão em experimentação.

\section{RESULTADOS E DISCUSSÃO}

De acordo com o roteiro de prática, a finalidade experimental era obter $450 \mathrm{~mL}$ de gás carbônico a partir da reação entre o carbonato de cálcio e ácido clorídrico e fazer uma comparação com os valores teóricos esperados. A Tabela 1 mostra os resultados experimentais obtidos e os parâmetros estatísticos calculados. 
Tabela 1. Resultados teóricos, experimentais e parâmetros estatísticos.

\begin{tabular}{|c|c|c|c|}
\hline TURMA & $\mathbf{V}_{\text {teórico }(\mathrm{mL})}$ & $\mathbf{V}_{\text {experimental (mL) }}$ & $\mathbf{E}_{\text {relativo (\%) }}$ \\
\hline $2 \mathrm{M} 1$ & 452,4 & 450 & $-0,53$ \\
\hline $2 \mathrm{M} 2$ & 450,7 & 450 & $-0,16$ \\
\hline $2 \mathrm{M} 3$ & 451,1 & 460 & 1,97 \\
\hline $2 \mathrm{M} 4$ & 451,7 & 445 & $-1,48$ \\
\hline $2 \mathrm{M} 5$ & 451,3 & 437 & $-3,17$ \\
\hline $2 \mathrm{~V} 1$ & 451,5 & 460 & 1,88 \\
\hline 2V2 & 450,5 & 435 & $-3,44$ \\
\hline 2V3 & 450,9 & 446 & $-1,09$ \\
\hline MÉDIA & 451,3 & 447,9 & $-0,75$ \\
\hline MÉDIA \pm SD & & $447,9 \pm 9,3$ & \\
\hline
\end{tabular}

Fonte: Dados da pesquisa.

As variáveis "V", "E" e "SD" da Tabela 1 representam o volume, o erro relativo e o desvio padrão da média, respectivamente. Os resultados experimentais obtidos foram satisfatórios e o maior erro relativo obtido foi de $-3,44 \%$, sendo erros de até $\pm 5 \%$ aceitáveis para este tipo de procedimento. O experimento foi repetido de maneira similar em oito turmas do $2^{\circ}$ ano e o erro relativo médio obtido foi de apenas $\quad-0,75 \%$, enquanto o desvio padrão da média foi de apenas $2,1 \%$. Quanto ao processo de construção do senso crítico dos alunos, pôde-se notar que houve reconhecimento de que é importante o desenvolvimento sustentável e é necessário reduzir a emissão de gases que aumentam a temperatura da Terra. A Figura 2 mostra algumas imagens do envolvimento dos alunos na aula. 


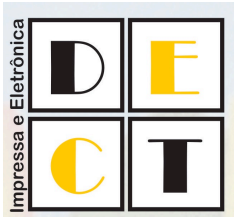

Figura 2. Envolvimento dos alunos na aula.
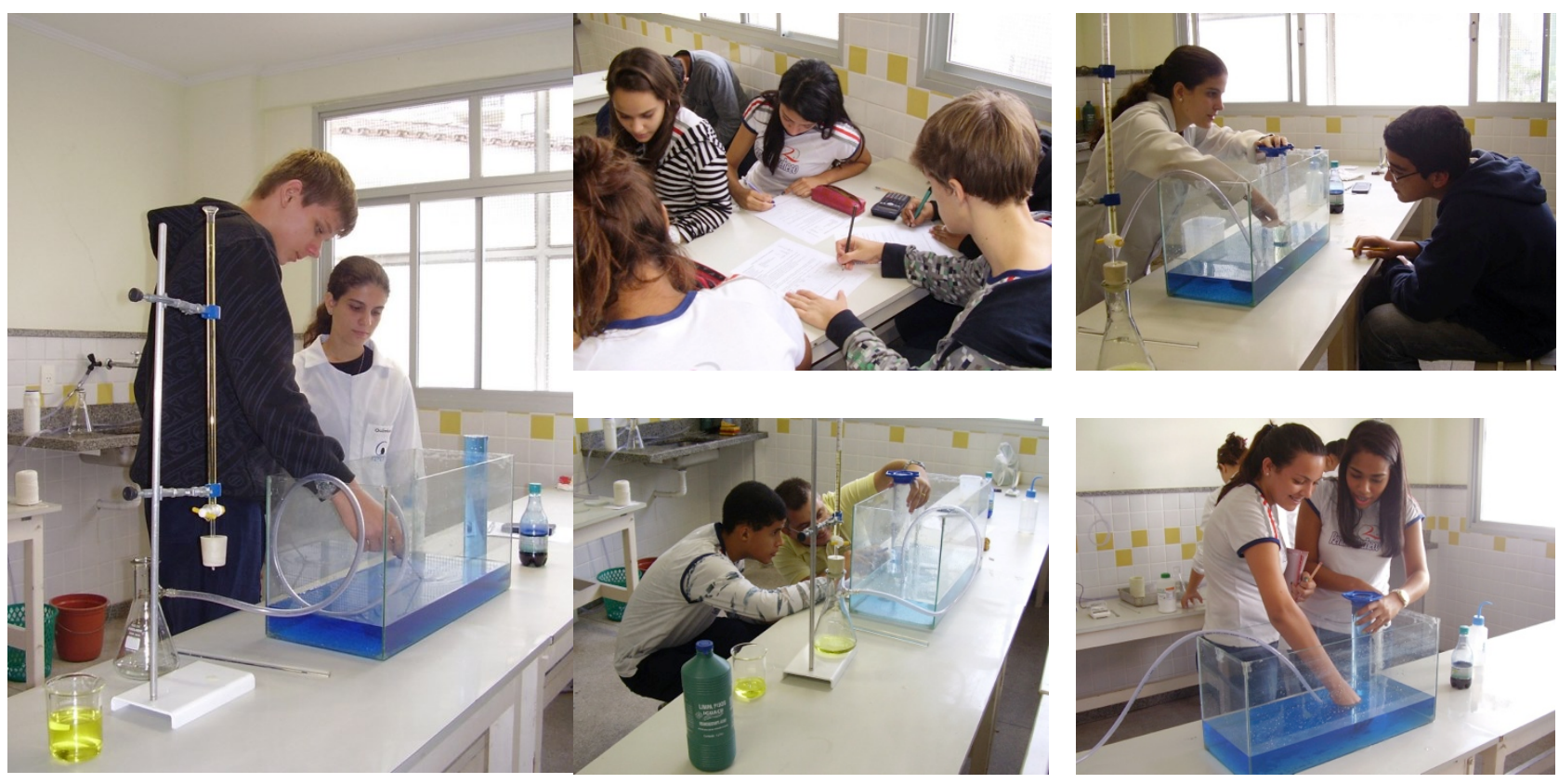

Fonte: Dados da pesquisa.

\section{CONSIDERAÇÕES FINAIS}

Como os alunos receberam o roteiro de prática com antecedência e foram orientados a estudá-lo antes da prática, a aula teve um rendimento satisfatório. O conhecimento teórico necessário ao entendimento da prática já havia sido estudado nas aulas demonstrativas a facilitaram a assimilação entre a teoria e a prática. Os alunos, em sua maioria, conseguiram efetuar de forma independente os cálculos necessários para tal assimilação, empregando corretamente as relações estequiométricas, a equação dos gases ideais e os cálculos estatísticos.

Os resultados experimentais foram reprodutíveis e aceitáveis em todas as turmas. O maior erro relativo obtido foi de $-3,4 \%$ e o desvio padrão da média foi de apenas 2,1\%. Quanto ao processo de construção do senso crítico dos alunos, pôde-se notar que houve reconhecimento de que é importante o desenvolvimento sustentável e é necessário reduzir a emissão de gases que aumentam a temperatura da Terra. 


\section{AGRADECIMENTOS}

Agradecemos ao apoio financeiro concedido pelo Programa Institucional de Bolsa de Iniciação à Docência - PIBID, da Coordenação de Aperfeiçoamento de Pessoal de Nível Superior - CAPES Brasil.

\section{REFERÊNCIAS}

CANTO, Eduardo Leite de. PERUZZO, Tito Miragaia. Química na Abordagem do Cotidiano. São Paulo: Moderna. 2012.

INMETRO. DOQ-DQUAL-006. Orientações para Adoção da NBR ISO/IEC 17025 - Pelos Laboratórios e Credenciados e Postulantes ao Credenciamento. 2001. 35 p.

REIS, Martha. Química Integral. Volume Único. São Paulo: FTD. 2012.

ROCHA, Marcelo Theoto. Aquecimento global e o mercado de carbono: uma aplicação do modelo CERT. São Paulo, 2003. 196 p. Tese de Doutorado, Escola Superior de Agricultura Luiz de Queiroz. Piracicaba, 2003.

SILVA, Rafaela C. O. Importância e Problemática da Utilização de Aulas Experimentais de Química no Ensino Médio no Município de Dois Vizinhos - Paraná. 2011. 35 f. Trabalho de Conclusão de Curso (Licenciatura em Química). Universidade Tecnológica Federal do Paraná. Pato Branco, 2011.

USBERCO, João. SALVADOR, Edgard. Química. Volume 1. São Paulo: Saraiva. 2012. 472 p. 